\title{
Review
}

\section{Postnatal Corticosteroids to Prevent or Treat Bronchopulmonary Dysplasia}

\author{
Lex W. Doyle ${ }^{a, b, c}$ \\ ${ }^{a}$ Newborn Research, The Royal Women's Hospital, Parkville, VIC, Australia; ${ }^{b}$ Departments of Obstetrics and \\ Gynaecology, and of Paediatrics, The University of Melbourne, Parkville, VIC, Australia; ${ }^{~}$ Clinical Sciences, \\ Murdoch Children's Research Centre, Parkville, VIC, Australia
}

\section{Keywords \\ Bronchopulmonary dysplasia $\cdot$ Corticosteroids $\cdot$ Inhaled . Systemic · Dexamethasone $\cdot$ Hydrocortisone $\cdot$ Cerebral palsy}

\begin{abstract}
Bronchopulmonary dysplasia (BPD) remains a major morbidity for infants born preterm. Postnatal corticosteroids might reduce the risk of developing $B P D$, or reduce its severity when it occurs, because of their powerful anti-inflammatory effects. However, corticosteroids have adverse effects, including on the developing brain. There have been numerous randomized clinical trials of corticosteroids given via various routes, of varying types, and started at different postnatal ages. There is some evidence that inhaled corticosteroids started earlier in the postnatal period may reduce BPD, but increase mortality. Inhaled corticosteroids started after the first week of age have little effect, but data are sparse. Systemic corticosteroids started in the first week after birth reduce BPD but increase cerebral palsy. Systemic corticosteroids started after the first week of age reduce both BPD and mortality, without evidence of long-term neurological harm. However, no studies have been powered to look for important adverse long-term neurological effects. Of the 2 system-
\end{abstract}

ic corticosteroids assessed, most effects relate to dexamethasone and not to hydrocortisone, but hydrocortisone in the first week after birth may reduce mortality, and is worthy of further study. There are limited data directly comparing inhaled versus systemic corticosteroids, with no evidence of superiority of one mode over the other. Corticosteroids instilled into the trachea using surfactant as a vehicle to distribute the drug through the lungs offer promise in preventing BPD. For current clinical practice, systemic corticosteroids should be avoided in the first week of life, and thereafter used only in infants at high risk of BPD.

() 2021 S. Karger AG, Basel

\section{Introduction}

Despite advances in obstetric and neonatal care, including the use of antenatal corticosteroids, exogenous surfactant, and more noninvasive respiratory support, bronchopulmonary dysplasia (BPD) remains a major complication of prematurity, affecting approximately $50 \%$ of infants born $<28$ weeks' gestation [1]. The pathology of BPD includes inflammation [2]. Because corticosteroids have powerful anti-inflammatory effects, sooner or later, they were bound to be used to try to prevent or 
Table 1. Routes of administration, numbers of studies, numbers of participants, types of corticosteroids, postnatal ages when treatment started, and overall quality of evidence of Cochrane reviews of postnatal corticosteroid treatments

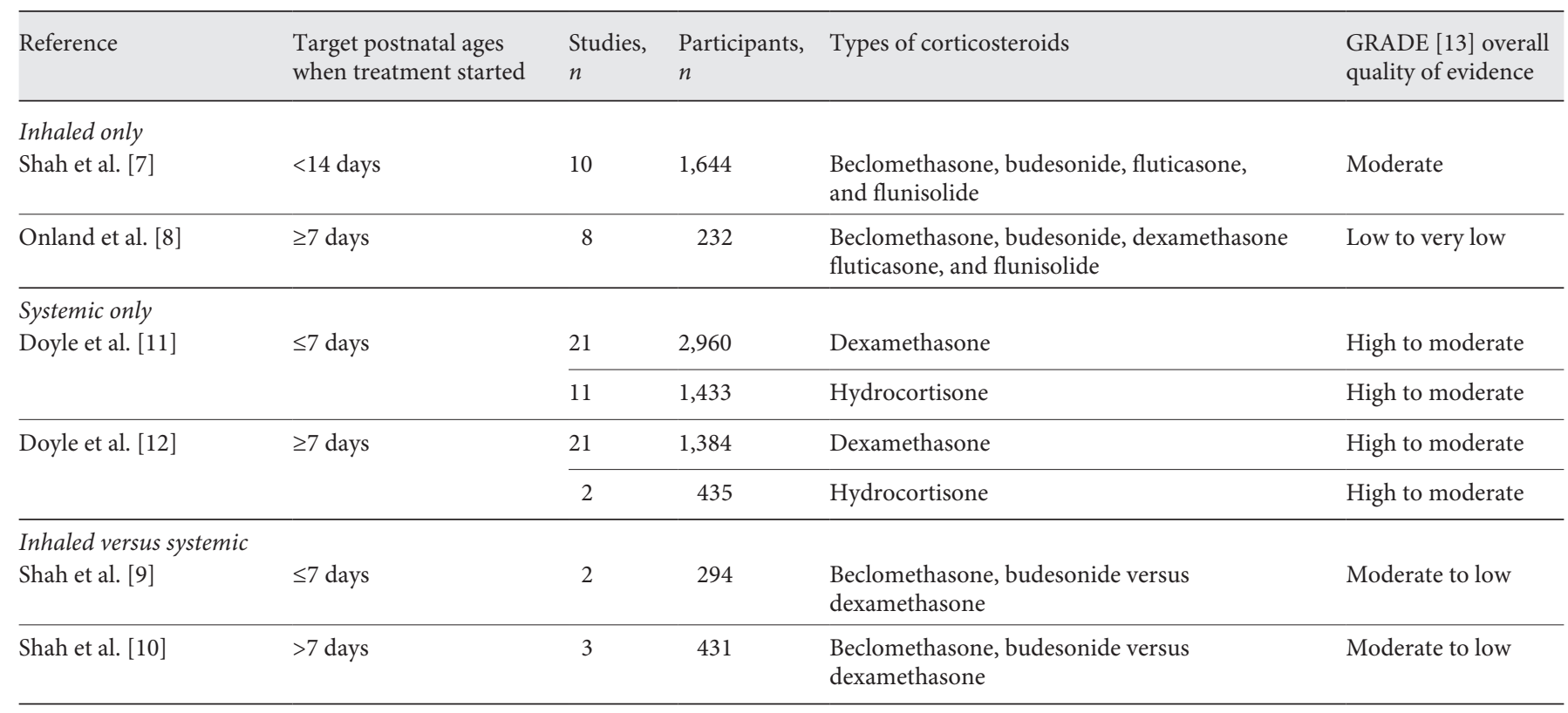

treat BPD. Although the first randomized controlled trial (RCT) of systemic corticosteroids after birth to treat breathing problems in preterm infants was reported almost 50 years ago [3], it was not until the 1980s that the first RCT of corticosteroids to treat BPD specifically was reported [4].

In the earliest RCTs to treat or prevent $\mathrm{BPD}$, the corticosteroids were given systemically $[5,6]$. As inhaled corticosteroids were developed for clinical use, they were evaluated in RCTs for BPD $[7,8]$. There have also been direct comparisons between inhaled and systemic corticosteroids $[9,10]$. A major rationale for inhaled corticosteroids over systemic corticosteroids was to avoid some of the complications of corticosteroids by concentrating the drug in the lung, minimizing exposure elsewhere in the body. Several different corticosteroids have been evaluated in RCTs, either dexamethasone or hydrocortisone for systemic administration, and even more for inhaled treatment (Table 1).

With $>80$ RCTs of corticosteroids to prevent or treat $\mathrm{BPD}$, enrolling $>9,000$ infants, corticosteroids are one of the most investigated medications in newborn medicine. After such intensive investigation, one might be forgiven for thinking that the answers about who and when to treat, and with what medication might be clear cut. Alas, that is not the case. The aim of this review was to consider the risks versus benefits of treatment with cortico- steroids after birth to prevent or treat BPD, allowing for the varying combinations of types of drug, and timing and routes of administration, with a focus on the major outcomes of BPD, mortality, and cerebral palsy.

\section{Data Sources}

There are multiple systematic reviews of postnatal corticosteroids in newborn infants in the Cochrane Library, including inhaled corticosteroids for either prevention of BPD [7] or for treatment of established lung disease [8], systemic corticosteroids where treatment started $\leq 7$ days [11] or $\geq 7$ days after birth [12], and inhaled versus systemic corticosteroids where the treatment started either $<14$ days after birth [9] or $\geq 7$ days after birth [10]. Details of the systematic reviews are shown in Table 1, along with the strength of the evidence to support the overall conclusions reported by the review authors using the GRADE system [13]. In addition to what is published in the Cochrane Library, there are 2 other systematic reviews of intratracheal corticosteroids, including where corticosteroid has been mixed with pulmonary surfactant and instilled into the trachea $[14,15]$. There are several key individual studies which will be discussed in more detail, all of which are included in one or more of the systematic reviews. 


\section{Major Outcomes}

The major outcomes in this review include BPD at 36 weeks' postmenstrual age, defined as oxygen dependency at 36 weeks in most, but not all studies, mortality at 36 weeks, and combined mortality or BPD at 36 weeks, mortality at the latest age reported, and, if data are available on long-term outcomes, cerebral palsy, mortality before follow-up, and combined mortality or cerebral palsy (Table 2). Some of the data in the existing systematic reviews have been updated where new information is available.

\section{Inhaled Corticosteroids}

\section{Early $(<14$ days) Inhaled Corticosteroids}

Inhaled corticosteroids lower the rates of BPD at 36 weeks (inhaled corticosteroids 20\% [132/649]; control $27 \%$ [170/636]), and combined mortality or BPD at 36 weeks (inhaled corticosteroids 35\% [227/649]; control $40 \%$ [256/636], Table 2). However, mortality by 36 weeks is not substantially different between the groups (inhaled corticosteroids 15\% [95/649]; control 14\% [86/636], Table 2).

Cerebral palsy is not substantially different between the groups (inhaled corticosteroids 6\% [37/574]; control $6 \%[34 / 553])$, but there is evidence that mortality before follow-up (inhaled corticosteroids 17\% [96/574]; control $12 \%$ [68/553]) and combined mortality or cerebral palsy (inhaled corticosteroids 23\% [133/574]; control 18\% [102/553]) are both higher in the inhaled corticosteroids group (Table 2).

The Neonatal European Study of Inhaled Steroids (NEuroSIS) trial [16] dominates the results. It recruited 856 infants born $23-27$ weeks' gestation aged $<12 \mathrm{~h}$ who were receiving respiratory support and who were randomized to either inhaled budesonide or control. The primary outcome of death or BPD at 36 weeks was $40 \%$ [175/437] in the budesonide group compared with $46 \%$ [194/419] in the control group (risk ratio [RR] 0.86, 95\% confidence interval $[\mathrm{CI}] 0.75,1.00, p=0.05)$. Although the rate of BPD was lower, there was an increase in mortality in the budesonide group by 36 weeks, which had increased further by 2 years of age (budesonide $(20 \%$ [82/419]; control $14 \%$ [58/400], RR 1.37, 95\% CI 1.01, $1.86, p=0.04)[17]$.

\section{Late ( $\geq 7$ days after Birth) Inhaled Corticosteroids}

The Cochrane review of late inhaled corticosteroids includes 8 RCTs and 232 participants. Only one trial con- tributed data on BPD at 36 weeks, only 3 had data for mortality at 36 weeks, and none reported long-term outcomes after discharge. Consequently, there are limited data for the major outcomes of interest in this review (Table 2).

\section{Systemic Corticosteroids}

\section{Early ( $\leq 7$ days after Birth) Systemic Corticosteroids}

There are 32 trials (21 dexamethasone and 11 hydrocortisone) randomizing 4,393 participants where systemic corticosteroids started within the first 6 days after birth have been compared with placebo or nothing [11]. Birth weights and gestational ages of the infants recruited vary widely, as do the regimens that have been assessed, with durations of treatment ranging from one dose up to 28 days, and total doses over a course of treatment from as low as 0.89 up to $14 \mathrm{mg} / \mathrm{kg}$ for dexamethasone.

Early systemic corticosteroids reduce the rates of BPD at 36 weeks (corticosteroids 25\% [513/2,090]; control $31 \%[640 / 2,077])$, and combined mortality or BPD by 36 weeks (corticosteroids 46\% [951/2,090]; control 51\% $[1,069 / 2,077])$, but do not affect mortality rates to 36 weeks alone (corticosteroids 20\% [396/1,961]; control $20 \%$ [393/1,953]) (Table 2) [11]. Most of the reduction of early systemic corticosteroids on BPD at 36 weeks arises from dexamethasone rather than hydrocortisone (dexamethasone 19\% [270/1,410]; control 27\% [372/1,381]; hydrocortisone 36\% [243/680]; control 39\% [268/696], Table 2), which is supported by $p=0.01$ for heterogeneity of effects of corticosteroids on BPD between the dexamethasone and hydrocortisone subgroups.

Both dexamethasone and hydrocortisone contribute to the reduction by early systemic corticosteroids on combined mortality or BPD at 36 weeks (dexamethasone $43 \%$ [600/1,410]; control 49\% [672/1,381]; hydrocortisone 51\% [350/680]; control 57\% [396/696]) (Table 2), with no substantial subgroup heterogeneity $(p=0.68)$. Early systemic corticosteroids overall do not reduce rates of mortality at the latest age reported (corticosteroids $22 \%$ [486/2,194]; control 23\% [506/2,179]) (Table 2). However, there is evidence for heterogeneity between dexamethasone and hydrocortisone subgroups $(p=0.05)$. Consequently, there is evidence that hydrocortisone reduces mortality at the latest reported age (hydrocortisone $18 \%$ [127/707]; control 22\% [163/727]), but not dexamethasone (dexamethasone 24\% [359/1,487]; control $24 \%$ [343/1,453]) (Table 2). 


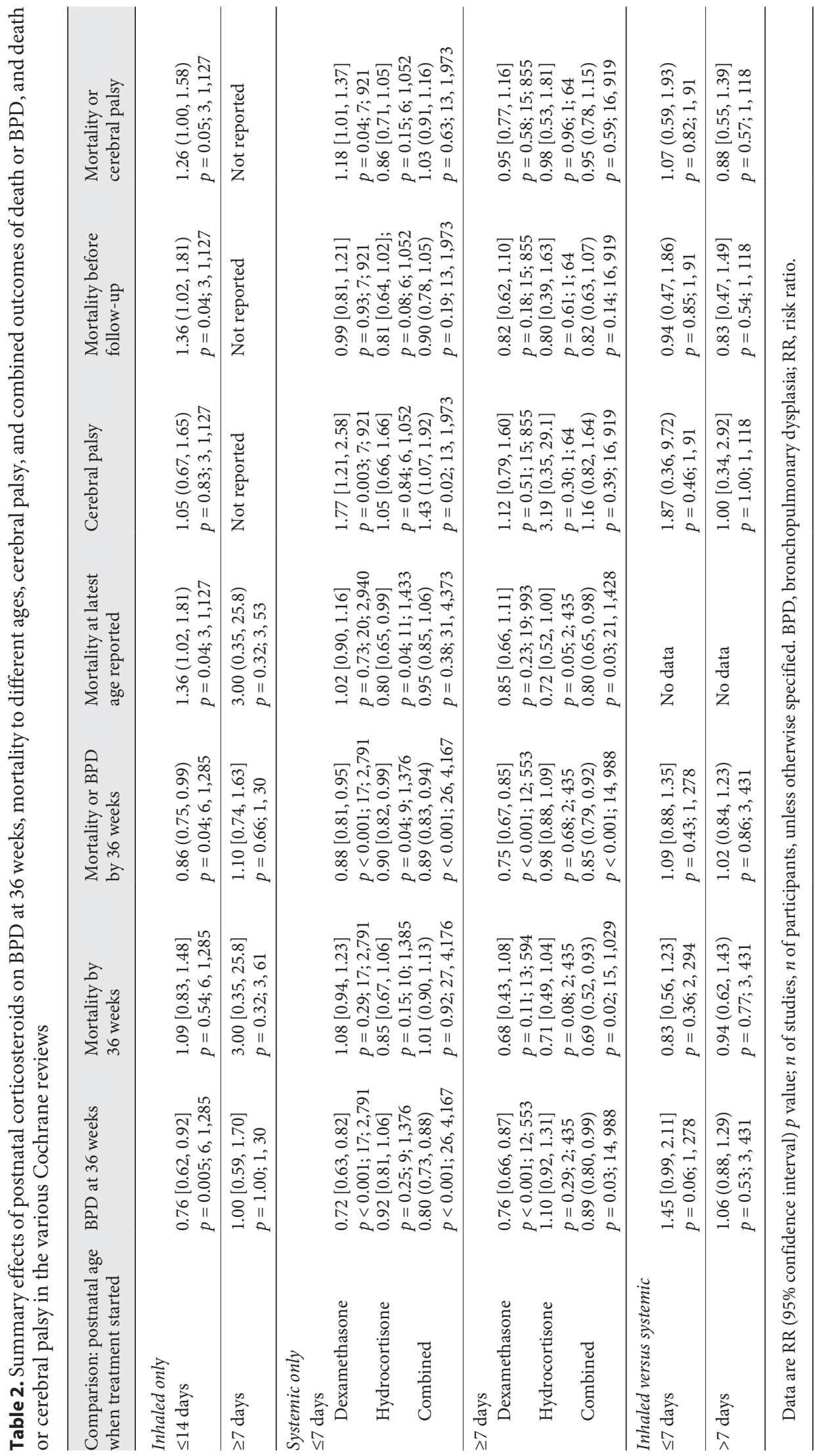


Early systemic corticosteroids increase the rates of cerebral palsy in later childhood (corticosteroids $11 \%$ [111/992]; control 7\% [73/981]; Table 2). Most of the effects on later cerebral palsy are due to dexamethasone (dexamethasone 16\% [76/472]; control 9\% [40/449]) (Table 2), although the interaction $p$ value is weak, at 0.09 . Although there is no evidence for an effect of early systemic corticosteroids overall on combined mortality or cerebral palsy, there is evidence of a subgroup interaction $(p=0.02)$. Early dexamethasone increases the rate of combined cerebral palsy or mortality (dexamethasone $45 \%$ [213/472]; control 38\% [172/449]), but hydrocortisone does not (Table 2).

One of the most influential trials in the early systemic corticosteroids review is the PREMILOC trial [18]. Baud et al. [18] recruited infants of 24-27 weeks' gestation who were $<24 \mathrm{~h}$ of age. Infants were randomly allocated to either a total of $8.5 \mathrm{mg} / \mathrm{kg}$ of hydrocortisone over 10 days, or identical placebo. The planned sample size was 786 infants, but the study stopped for logistical reasons with 521 infants recruited after almost 6 years, two-thirds of the original sample size. Hydrocortisone reduced the rate of the primary end point of BPD or mortality at 36 weeks (hydrocortisone 40\% [102/255]; control 49\% [130/266], RR 0.82 ; 95\% CI $0.67-0.99 ; p=0.04$ ). At follow-up at 22 months' corrected age, rates of cerebral palsy were similar (hydrocortisone 6\% [12/194]; control 5\% [10/185]) [19].

\section{Other Effects of Early Systemic Corticosteroids}

Other benefits of early systemic corticosteroids include lower rates of extubation failure, patent ductus arteriosus, and severe retinopathy of prematurity [11]. However, there are also adverse effects, including gastrointestinal bleeding, intestinal perforation, hyperglycaemia, hypertension, hypertrophic cardiomyopathy, and growth failure [11]. Most of the other benefits and risks of early systemic corticosteroids relate to dexamethasone but not to hydrocortisone. The exception is gastrointestinal perforation, which is more common with both types of corticosteroid compared with control, with no evidence for heterogeneity between subgroups $(p=0.61)$ [11].

\section{Limitations of Early Systemic Corticosteroid Trials}

The major limitation is that all studies reporting longterm outcomes in early childhood were not powered to find differences in neurodevelopmental outcomes, and hence data on the true long-term benefits versus risks cannot be fully determined. Most studies focused on short-term outcomes in the neonatal period, such as ventilator- or oxygen dependence.
Late ( $\geq 7$ days after Birth) Systemic Corticosteroids

There are 23 trials (21 dexamethasone and 2 hydrocortisone) recruiting 1,817 infants where systemic corticosteroids started on or after day 7 after birth have been compared with placebo or nothing [12]. Most infants were intubated and ventilator dependent upon enrolment and were mostly of birth weight $<1,500 \mathrm{~g}$. Durations of treatment ranged from 2 up to 42 days, and total doses in the initial course of treatment ranged from 0.65 up to $8.5 \mathrm{mg} / \mathrm{kg}$ for dexamethasone.

Late systemic corticosteroids reduce the rates of BPD at 36 weeks (corticosteroids 53\% [263/498]; control 59\% [291/490]), mortality to 36 weeks (corticosteroids $12 \%$ [64/515]; control 18\% [90/514]), and combined mortality or BPD by 36 weeks (corticosteroids 65\% [326/498]; control 77\% [378/490]) (Table 2) [12].

All of the effects of late systemic corticosteroids on BPD at 36 weeks arise from use of dexamethasone rather than hydrocortisone (Table 2), with $p=0.001$ for heterogeneity of effects of corticosteroids on BPD between the dexamethasone and hydrocortisone subgroups (dexamethasone 50\% [143/286]; control 66\% [176/267]; hydrocortisone 57\% [120/212]; control 51\% [115/223]). Similarly, most of the effects of late systemic corticosteroids on combined mortality or BPD by 36 weeks arise from the use of dexamethasone rather than hydrocortisone (Table 2), with $p=0.001$ for heterogeneity for the differential effect between subgroups (dexamethasone 59\% [170/286]; control 79\% [210/267]; hydrocortisone $74 \%$ [156/212]; control 75\% [168/223]). There is little evidence for subgroup heterogeneity between dexamethasone and hydrocortisone subgroups for effects of either corticosteroid on mortality to 36 weeks $(p=0.82)$.

Late systemic corticosteroids reduce the rates of mortality at the latest age reported (corticosteroids 18\% [131/714]; control 23\% [161/714]) (Table 2). Although it appears that hydrocortisone reduces mortality at the latest reported age (hydrocortisone 21\% [45/212]; control $30 \%$ [66/223]) more than dexamethasone (dexamethasone 17\% [86/502]; control 24\% [95/491]) (Table 2), there is little evidence for heterogeneity between dexamethasone and hydrocortisone subgroups $(p=0.42)$.

There is little evidence that late systemic corticosteroids affect the rates of cerebral palsy in later childhood in those randomized (late systemic corticosteroids 13\% [61/463]; control 11\% [52/456]) (Table 2). Only one of 16 trials contributing to this analysis was assessing hydrocortisone, so there was no attempt to look for subgroup differences between dexamethasone and hydrocortisone on cerebral palsy [12]. Similarly, rates of combined death 
or cerebral palsy at follow-up were similar between late corticosteroid and control groups (late systemic corticosteroids 31\% [142/463]; control 32\% [145/456]) (Table 2).

One of the more important trials in the late systemic corticosteroids group is the SToP-BPD trial. Onland et al. [20] recruited 372 infants born either $<30$ weeks' gestation or $<1,250 \mathrm{~g}$ birth weight who were ventilator dependent between 7 and 14 days of age. Infants were randomly allocated to either a total of $72.5 \mathrm{mg} / \mathrm{kg}$ of hydrocortisone over 22 days or identical placebo. The primary end point was mortality or BPD at 36 weeks, in which they found no important difference (hydrocortisone $71 \%$ [128/181] vs. control 74\% [140/190], RR 0.87; 95\% CI $0.54-1.38 ; p=0.54$ ). Although only one of 14 trials contributing data to the combined end point of mortality or BPD at 36 weeks, it comprises $36 \%$ of the weight to that outcome. Follow-up at 2 years is planned for the SToPBPD study.

More information on long-term outcomes of hydrocortisone after the first week of life will also soon be available from a trial in the USA which has completed recruitment (https://clinicaltrials.gov/ct2/show/NCT01353313). A 10-day tapering course of $18 \mathrm{mg} / \mathrm{kg}$ of hydrocortisone has been given to ventilator-dependent infants $<30$ weeks, aged 14-28 days. The study is powered for its co-primary outcomes of survival free of moderate-severe BPD and survival without moderate-severe neurodevelopmental impairment at 18-22 months.

\section{Other Effects of Late Systemic Corticosteroids}

Other benefits of late systemic corticosteroids include lower rates of extubation failure, less need for open-label corticosteroids, and fewer infants needing to be discharged home of oxygen [12]. Against that, late corticosteroids cause more hyperglycaemia, glycosuria, hypertension, cardiomyopathy, and severe retinopathy of prematurity [12]; these latter problems resolve after the corticosteroids are stopped. Other outcomes in later childhood including respiratory health or function, blood pressure, or growth are similar between groups, with the exception of improved expiratory airflow in the corticosteroid group, but are based on few studies with not many participants [12].

\section{Limitations of Late Systemic Corticosteroid Trials}

As with the early systemic corticosteroid trials, most of the studies reporting long-term outcomes were not powered to detect clinically important neurodevelopmental differences between the corticosteroid and control groups.

Postnatal Corticosteroids and BPD

\section{Inhaled versus Systemic Corticosteroids}

There are 2 Cochrane reviews where inhaled and systemic corticosteroids have been compared $[9,10]$. Both reviews are characterized by small numbers of studies and participants, complex study designs, and limited longterm data.

\section{Early Inhaled versus Systemic Corticosteroids}

There were no clear differences between early inhaled corticosteroids and early systemic corticosteroids for BPD, mortality, or cerebral palsy (Table 2). Differences in other short-term outcomes in the Cochrane review include longer durations of assisted ventilation by an average of almost 4 days and of oxygen therapy by 11 days, but less hyperglycaemia with inhaled corticosteroids [9].

\section{Later Inhaled versus Systemic Corticosteroids}

There was no evidence for any benefit or harm of inhaled corticosteroids compared with systemic corticosteroids for BPD, mortality, or cerebral palsy (Table 2). Other short-term complications of corticosteroids were not substantially different between the groups in the Cochrane review [10].

\section{Intratracheal Corticosteroids Administered with a Surfactant}

Of the 2 systematic reviews of intratracheal corticosteroids administered with a surfactant, the first includes only 2 RCTs [14], with the second review adding 3 more RCTs, and several other RCTs of inhaled corticosteroids [15]. In the later and hence more up-to-date review, the risk of BPD (not defined) was lower in the corticosteroidsurfactant groups (23\% [60/260]) compared with the surfactant-only group (42\% [117/279]; RR 0.57; 95\% CI $0.44-0.73 ; p<0.001 ; 5$ studies, 539 participants) [15]. The risk of mortality (age not defined) was lower in the corticosteroid-surfactant groups (13\% [27/206]) versus the surfactant-only group (20\% [42/205]; RR $0.64 ; 95 \%$ CI $0.41-0.99 ; p=0.04 ; 3$ studies, 411 participants). The results of this review are dominated by the larger of 2 studies from researchers in Taiwan, which is also the only study to report follow-up data to 30 months for 172 survivors, with no substantial differences found in neurodevelopmental outcomes [21]. 


\section{Clinical Implications}

\section{Inhaled Corticosteroids}

Although there is some evidence for reducing rates of reintubation with early ( $<14$ days) inhaled corticosteroids [7], on balance, the reduction in BPD at 36 weeks is outweighed by the increase in mortality when it comes to considering early inhaled corticosteroids for routine clinical use. The lack of data for inhaled corticosteroids after the first week means they cannot be recommended for routine clinical practice.

\section{Early ( $<7$ days) Systemic Corticosteroids}

There are benefits and harms from early systemic corticosteroids in the short term, including a possible reduction in mortality with early systemic hydrocortisone, but also long-term harm, in particular an increase in cerebral palsy with early systemic dexamethasone [11]. Early systemic corticosteroids to prevent BPD or other lung injury should not be used in routine clinical practice. More RCTs of early hydrocortisone would be worthwhile, with long-term survival free of neurodevelopmental disability as the primary end point.

\section{Late Systemic Corticosteroids}

The current evidence favours dexamethasone starting from day 7 or later after birth in infants at high risk of developing BPD [22], but this may change when long-term neurodevelopmental data are available from 2 large trials of systemic hydrocortisone which have completed recruitment.

\section{Inhaled versus Systemic Corticosteroids}

The lack of evidence for any benefits or harms of inhaled corticosteroids over systemic corticosteroids for any important outcomes means that one cannot be recommended over the other for treatment at any age. Future studies comparing inhaled with systemic corticosteroids should have simpler study designs and focus on major clinically important outcomes as their end points.

\section{Intratracheal Administration Combined with \\ Surfactant}

Intratracheal administration of corticosteroids with surfactant looks promising, but the limited follow-up data from only one study thus far are not enough to recommend this therapy for routine clinical use just yet. More information should soon be available from several other RCTs that are either recruiting or planned (ACTRN12617000322336; https://clinicaltrials.gov/ct2/show/NCT03521063; https://
clinicaltrials.gov/ct2/show/NCT03275415) to help to guide clinical practice regarding intratracheal administration of corticosteroids in surfactant.

\section{Conclusions}

The role of corticosteroids in preventing or treating $\mathrm{BPD}$ is not clearly established. On balance, the risks versus benefits are most favourable for systemic corticosteroids after the first week of age for infants at the highest risk of BPD [22], which would be intubated infants. Evidence still exists for using dexamethasone, but this may be surpassed when long-term neurodevelopmental data are available from 2 large trials of systemic hydrocortisone which have completed recruitment. Whichever drug is used, its dose and duration should be minimized. More research is required into the role of corticosteroids in non-mechanically ventilated infants, corticosteroid-surfactant mixtures, hydrocortisone in the first week after birth, and determining outcomes into school-age and beyond for as many infants randomized as possible.

\section{Conflict of Interest Statement}

Dr. Doyle is the first author of 2 relevant reviews in the Cochrane Library on the topic of systemic corticosteroids to prevent or treat bronchopulmonary dysplasia. He was the chief investigator on the DART study - a randomized controlled trial of postnatal dexamethasone in ventilator-dependent infants. He is a chief investigator of the current PLUSS study, a current randomized trial of budesonide in surfactant in infants $<28$ weeks' gestation.

\section{Funding Sources}

Supported in part by Project Grant No. 108700, Program Grant No. 606789, and Centre of Research Excellence Grants No. 1060733 and No. 1153176 from the National Health and Medical Research Council (NHMRC) of Australia. The funding source had no role in the collection, analysis, or interpretation of data, or in the writing of the manuscript.

\section{Author Contributions}

Dr. Doyle conceived, researched, and wrote the article. 


\section{References}

1 Doyle LW, Ranganathan S, Cheong JLY. Ventilation in preterm infants and lung function at 8 years. N Engl J Med. 2017;377(377): 1601-2.

2 Jobe AH, Bancalari E. Bronchopulmonary dysplasia. Am J Respir Crit Care Med. 2001; 163(7):1723-9.

3 Baden M, Bauer CR, Colle E, Klein G, Taeusch HW Jr, Stern L. A controlled trial of hydrocortisone therapy in infants with respiratory distress syndrome. Pediatrics. 1972;50(50): 526-34.

4 Avery GB, Fletcher AB, Kaplan M, Brudno DS. Controlled trial of dexamethasone in respirator-dependent infants with bronchopulmonary dysplasia. Pediatrics. 1985;75(1): 106-11.

5 Doyle LW, Cheong JL, Ehrenkranz RA, Halliday HL. Early ( $<8$ days) systemic postnatal corticosteroids for prevention of bronchopulmonary dysplasia in preterm infants (review). Cochrane Database Syst Rev. 2017;10: CD001146.

6 Doyle LW, Cheong JL, Ehrenkranz RA, Halliday HL. Late ( $>7$ days) systemic postnatal corticosteroids for prevention of bronchopulmonary dysplasia in preterm infants (review). Cochrane Database Syst Rev. 2017;10: CD001145.

7 Shah VS, Ohlsson A, Halliday HL, Dunn M. Early administration of inhaled corticosteroids for preventing chronic lung disease in ventilated very low birth weight preterm neonates. Cochrane Database Syst Rev. 2017;1: CD001969.

8 Onland W, Offringa M, van Kaam A. Late ( $\geq 7$ days) inhalation corticosteroids to reduce bronchopulmonary dysplasia in preterm infants. Cochrane Database Syst Rev. 2017;8: CD002311.
9 Shah SS, Ohlsson A, Halliday HL, Shah VS. Inhaled versus systemic corticosteroids for preventing bronchopulmonary dysplasia in ventilated very low birth weight preterm neonates. Cochrane Database Syst Rev. 2017; 10(10):CD002058.

10 Shah SS, Ohlsson A, Halliday HL, Shah VS. Inhaled versus systemic corticosteroids for the treatment of bronchopulmonary dysplasia in ventilated very low birth weight preterm infants. Cochrane Database Syst Rev. 2017;10: CD002057.

11 Doyle LW, Cheong JL, Manley B, Hay S, Halliday HL. Early ( $<7$ days) systemic postnatal corticosteroids for prevention of bronchopulmonary dysplasia in preterm infants (review). Cochrane Database Syst Rev. Forthcoming. 2021.

12 Doyle LW, Cheong JL, Manley B, Hay S, Halliday HL. Late ( $>=7$ days) systemic postnatal corticosteroids for prevention of bronchopulmonary dysplasia in preterm infants (review). Cochrane Database Syst Rev. Forthcoming. 2021.

13 Group GW. GRADE handbook for grading quality of evidence and strength of recommendations. In: Schünemann H, Brożek J, Guyatt G, Oxman A, editors; 2013. Updated 2013 Oct. Available from: https://gdt. gradepro.org/app/handbook/handbook. html.

14 Venkataraman R, Kamaluddeen M, Hasan SU, Robertson HL, Lodha A. Intratracheal administration of budesonide-surfactant in prevention of bronchopulmonary dysplasia in very low birth weight infants: a systematic review and meta-analysis. Pediatr Pulmonol. 2017;52(52):968-75.

15 Zhong YY, Li JC, Liu YL, Zhao XB, Male M, Song DK, et al. Early intratracheal administration of corticosteroid and pulmonary surfactant for preventing bronchopulmonary dysplasia in preterm infants with neonatal respiratory distress syndrome: a meta-analysis. Curr Med Sci. 2019;39(3):493-9.
16 Bassler D, Plavka R, Shinwell ES, Hallman M Jarreau PH, Carnielli V, et al. Early inhaled budesonide for the prevention of bronchopulmonary dysplasia. N Engl J Med. 2015;373: 1497-506.

17 Bassler D, Shinwell ES, Hallman M, Jarreau PH, Plavka R, Carnielli V, et al. Long-term effects of inhaled budesonide for bronchopulmonary dysplasia. N Engl J Med. 2018;378: 148-57.

18 Baud O, Alberti C, Mohamed D, Watterberg $\mathrm{K}$. Low-dose hydrocortisone in extremely preterm infants: authors' reply. Lancet. 2016; 388(388):1158-9.

19 Baud O, Trousson C, Biran V, Leroy E, Mohamed D, Alberti C, et al. Association between early low-dose hydrocortisone therapy in extremely preterm neonates and neurodevelopmental outcomes at 2 years of age. JAMA. 2017;317(13):1329-37.

20 Onland W, Cools F, Kroon A, Rademaker K, Merkus MP, Dijk PH, et al. Effect of hydrocortisone therapy initiated 7 to 14 days after birth on mortality or bronchopulmonary dysplasia among very preterm infants receiving mechanical ventilation: a randomized clinical trial. JAMA. 2019;321(321):354-63.

21 Yeh TF, Chen CM, Wu SY, Husan Z, Li TC, Hsieh WS, et al. Intratracheal administration of budesonide/surfactant to prevent bronchopulmonary dysplasia. Am J Respir Crit Care Med. 2016;193(1):86-95.

22 Doyle LW, Halliday HL, Ehrenkranz RA, Davis PG, Sinclair JC. An update on the impact of postnatal systemic corticosteroids on mortality and cerebral palsy in preterm infants: effect modification by risk of bronchopulmonary dysplasia. J Pediatr. 2014;165(165): 1258-60. 